\title{
A Case Report on Recurrent Merkel Cell Carcinoma with Metastasis to Colon Treated with Pembrolizumab
}

\author{
Shobha M*', Baniya SS², Sumit G³ ${ }^{3}$, Sunil S ${ }^{3}$, Yadav \\ $\mathbf{N}^{4}$ and J oyson $\mathbf{P}^{5}$ \\ ${ }^{1}$ Department of Internal medicine, Guthrie Robert Packer \\ Hospital, Sayre, PA, USA \\ ${ }^{2}$ Department of Internal Medicine, State College, PA, USA \\ ${ }^{3}$ Department of Medicine, Universal College of medical \\ sciences, Bhairahawa, Nepal \\ ${ }^{4}$ Department of Medicine, B.P. Koirala Institute of Health \\ Sciences, Dharan, Nepal \\ ${ }^{5}$ Department of Hemato-oncology, Guthrie Robert Packer \\ Hospital, Sayre, PA, USA \\ *Corresponding author: Mandal Shobha, Department \\ of Internal medicine, Guthrie Robert Packer Hospital, 126 \\ hemlock road, apt 6, Sayre, PA 18840, USA
}

Received: April 14, 2021; Accepted: May 11, 2021; Published: May 18, 2021

\begin{abstract}
Merkel Cell Carcinoma (MCC) is a cutaneous neuroendocrine cancer with a poor prognosis. It is characterized by a high rate of recurrence and metastases to regional as well as distant sites. Clinically, MCC often manifests as a single painless, hard nodule in the sun-exposed area. The diameter is often $<2 \mathrm{~cm}$, but in few cases, it can be $>2 \mathrm{~cm}$, with rapid growth and metastases to lymph nodes. MCC carcinoma is diagnosed in the advanced stage because of its resemblance with other skin cancers. Because of the aggressive nature of this cancer, the overall prognosis is found to be poor. In this case report, we report a 71-year-old gentle male who presented with a painless mass in the right axilla three years ago, was diagnosed with MCC of the skin and was treated with surgical removal of the mass together with radiation therapy. Two years later, the follow-up visit restaging PET CT scan was done and was found to have a new intense uptake in the soft tissue nodule adjacent to the proximal sigmoid colon. Biopsy confirmed the metastasis of MCC to the colon. We are reporting an uncommon location of MCC metastasis to the intestine for which he got treated with immunotherapy.
\end{abstract}

Keywords: Merkel cell carcinoma; MCC; Immunotherapy; Pembrolizumab

\section{Introduction}

Merkel Cell Carcinoma (MCC) is rare and clinically aggressive skin cancer, with five years survival of nearly $18 \%$ for advanced-stage MCC [1]. MCC's annual incidence is about 3 per 1000000 worldwide [2] and 0.79 per 100,000 in the United States [3]. It presents as a rapidly growing, firm, red to violaceous nodule [4]. Common risk factors associated with MCC are old age, fair-skin, immunosuppression, ultraviolet light exposure, polyomavirus infection [5,6]. It has a high propensity for recurrence because of metastases to distant sites. It rarely metastasizes to the gastrointestinal tract. Recently Immunotherapy like pembrolizumab has shown promising effects in the treatment of MCC. We report a 71-year-old male diagnosed with MCC of skin and was initially treated with surgical removal and radiation therapy. Later, on follow-up restaging, he was found to have a tumor recurrence in the colon. He got treated with Pembrolizumab.

\section{Case Presentation}

A 71-year-old white American male with a past medical history of hypertension, atrial fibrillation, cerebrovascular accident, coronary artery disease, and oxygen-dependent chronic obstructive pulmonary disease presented to the primary care physician with a painless mass in the right axilla three years ago. The mass was gradually increasing in size over one year and was non-tender without any pus or redness. He denied any surgery, trauma, insect bite, fever, or any history of any neoplastic process in the past. Family history was positive for some unknown cancer in grandfather. On exam, $1 \times 1 \mathrm{~cm}$ mass was present in the right axillary region. On a detailed skin exam, there was no evidence of any sun-induced skin lesion or lymphadenopathy. Laboratories workup was within normal limits. Right axillary ultrasound showed an irregular hypoechoic mass measuring 4.7 x 5.9

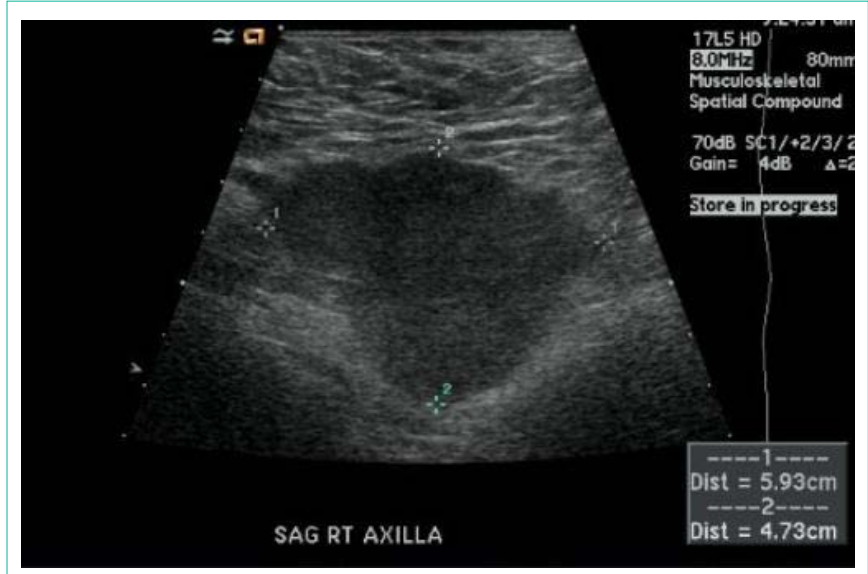

Figure 1: Right axillary ultrasound showed an irregular hypoechoic mass measuring $4.7 \times 5.9 \mathrm{~cm}$.

$\mathrm{cm}$ (Figure 1).

Positron Emission, Tomography/CT scan, showed a right upper lobe $8 \mathrm{~mm}$ nodule, the focus of conglomerate right axillary lymph node with SUV of 14, $1.6 \mathrm{~cm}$ enlarged left parotid gland (Figure 2).

He underwent a biopsy of the mass positive for high-grade neuroendocrine carcinoma with morphological findings of Merkel cell carcinoma (Figure 2-7).

Immunohistochemistry was positive for CK20, CD56, Synaptophysin, and Chromogranin but was negative for CK7, S100, SOX10.

He underwent surgical removal of the axillary mass with wide
Ann Hematol Oncol - Volume 8 Issue 6 - 2021

ISSN : 2375-7965 | www.austinpublishing group.com

Shobha et al. @ All rights are reserved
Citation: Shobha M, Baniya SS, Sumit G, Sunil S, Yadav N and Joyson P. A Case Report on Recurrent Merkel Cell Carcinoma with Metastasis to Colon Treated with Pembrolizumab. Ann Hematol Oncol. 2021; 8(6): 1348. 


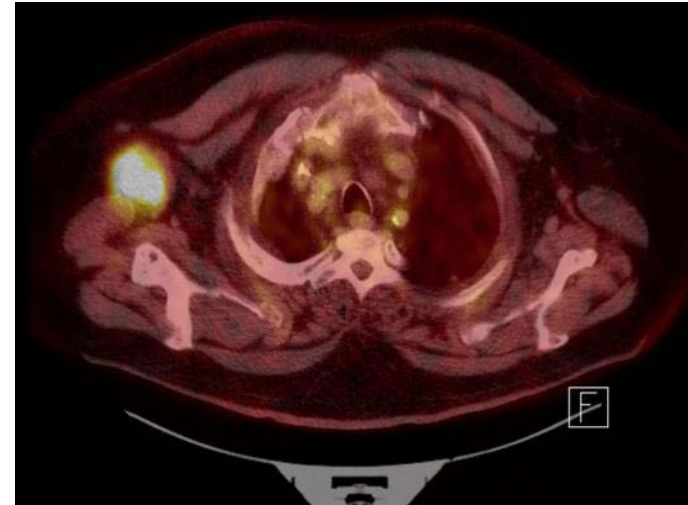

Figure 2: Tomography/CT scan, showed a right upper lobe $8 \mathrm{~mm}$ nodule, the focus of conglomerate right axillary lymph node with SUV of $14,1.6 \mathrm{~cm}$ enlarged left parotid gland.

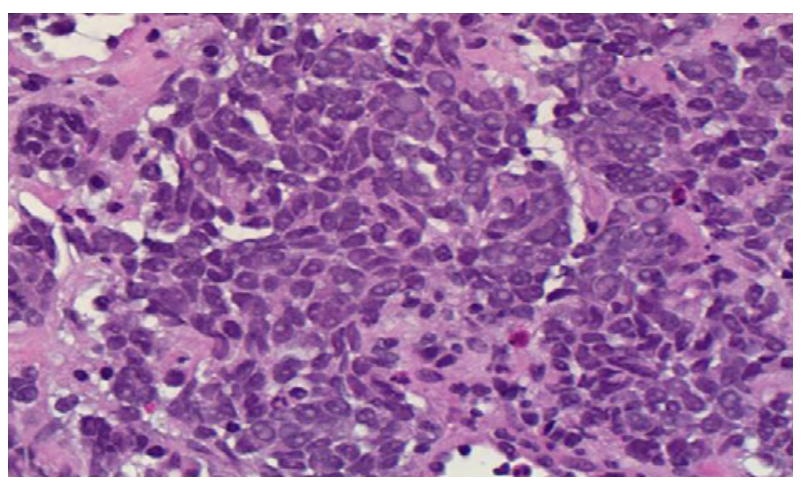

Figure 3: Immunohistopathology showing findings suggestive of MCC. Round to spindle-shaped blue tumor cells with salt and pepper chromatin pattern and inconspicuous nucleoli; H \& E X400.



Figure 4: Immunohistochemistry showing tumor cell positive for pan keratin (perinuclear dot pattern) $\times 400$.

excision of the skin and got treated with radiation therapy for one dose. On the follow-up visit one year later, he denied any active complaint. He underwent a restaging PET CT scan that did not show abnormal FDG uptake in the right axilla, with no new FDG avid disease sites. Two years later, a repeat PET scan showed a slight interval increase in the degree of uptake in the subpleural nodule in the lateral right upper lobe measuring $1.4 \times 0.8 \mathrm{~cm}$, a newly intense uptake soft tissue nodule situated adjacent to the proximal sigmoid

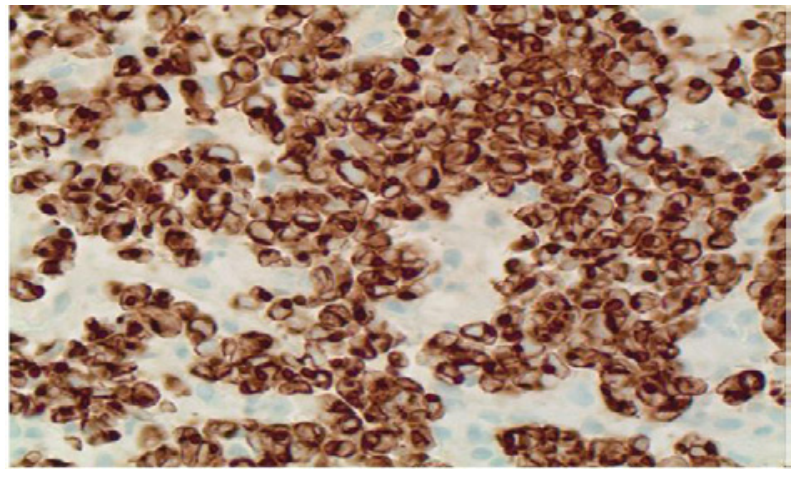

Figure 5: Immunohistochemistry showing tumor cell positive for CK20 (perinuclear dot pattern) $\times 400$

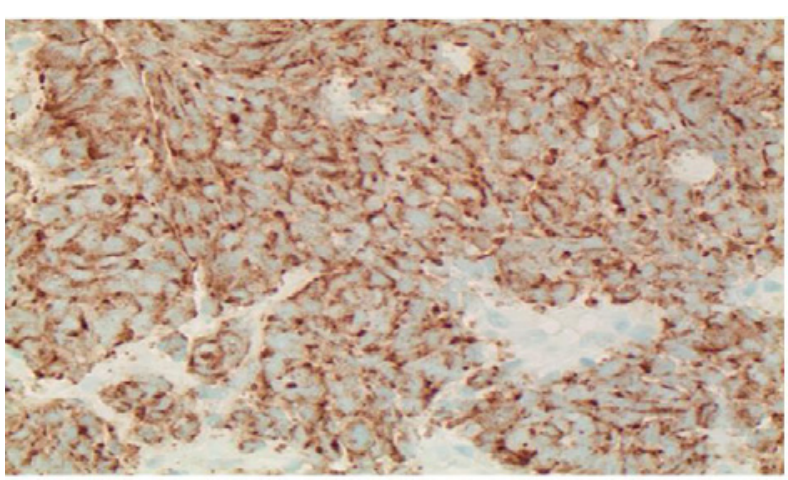

Figure 6: Immunohistochemistry showing tumor cell positive for Chromogranin $\times 400$.

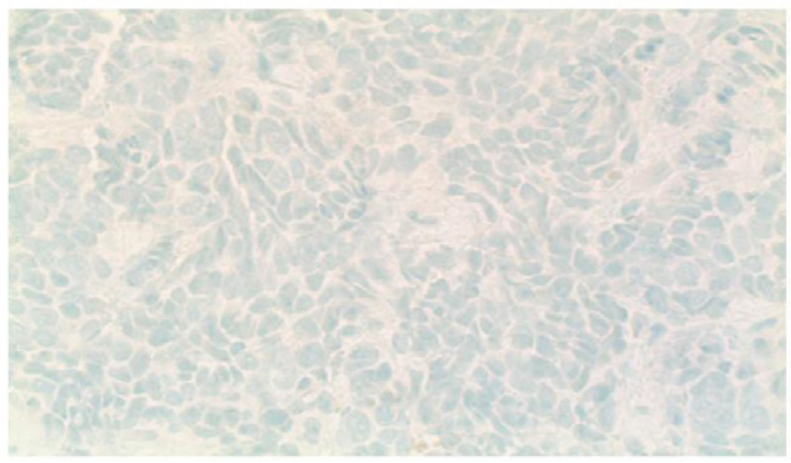

Figure 7: Immunohistochemistry showing tumor cell negative for CK7 x400.

colon (Figure 8).

On the same visit, he complained of an isolated episode of rectal bleeding a few days, denied any other complaint. Laboratory work showed low hemoglobin of $9.7 \mathrm{mg} / \mathrm{dl}$, and other labs were stable. He underwent colonoscopy, which showed a partially obstructing tumor in the proximal ascending colon. Upper endoscopy showed mildly erythematous mucosa without bleeding in the gastric antrum. Biopsy of the ascending colon tumor was positive for small cell neuroendocrine carcinoma with a high KI-67 proliferation index of $50-60 \%$. 


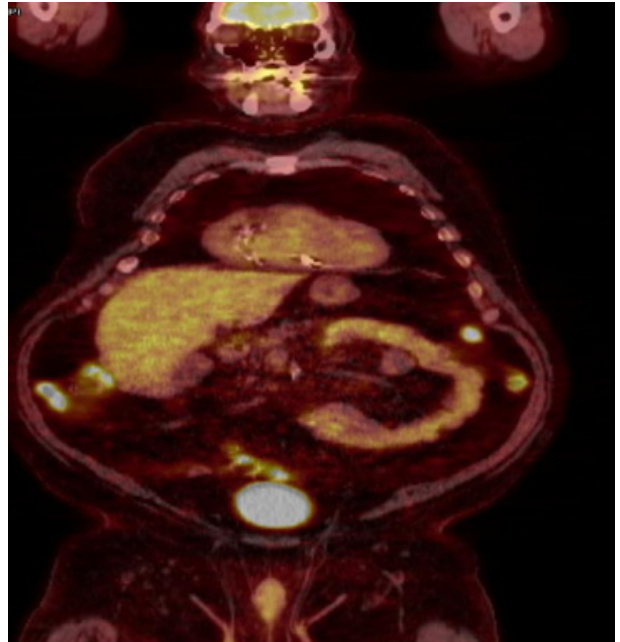

Figure 8: PET scan showed a slight interval increase in the degree of uptake in the subpleural nodule in the lateral right upper lobe measuring $1.4 \times 0.8 \mathrm{~cm}$, a newly intense uptake soft tissue nodule situated adjacent to the proximal sigmoid colon.

As the patient had a recurrence of a small cell neuroendocrine tumor, he started treatment with Pembrolizumab 200mg every three weeks. He tolerated the therapy well and was monitored with restaging computed tomography chest abdomen and pelvis every six months. He successfully completed 22 cycles of Pembrolizumab. He complained of a new-onset sob recently on his follow-up visit, was requiring 3 liters of oxygen. CT of the chest showed enlarging bilateral pleural effusions. He underwent a thoracentesis with the removal of approximately $1 \mathrm{~L}$ of transudative fluid. Pleural fluid analysis was negative for any malignant cell. He reported improvement in his symptoms of shortness of breath. The radiation oncologist recommended no need for radiation therapy and continued the scheduled pembrolizumab and laboratory workup monitoring.

\section{Discussion}

Merkel cell carcinoma is a rare, clinically aggressive malignant neuroendocrine neoplasm of the skin. It most commonly arises from the skin's dermis but rarely can appear outside the skin [7]. It is most of the time locally invasive but can frequently metastasize to lymph nodes, liver, lungs, bone, and brain. MCC was first described as trabecular cell carcinoma by Toker in 1972 [8]. MCC most commonly affects the older white male population, but females can also be affected. The most affected areas are head and neck (50\%), upper and lower limbs (35\%-40\%), <10\% in the trunk but can rarely involve the vulva, penis, pharynx, oral and nasal mucosa [9]. MCC's risk factors are old age, Ultraviolet (UV) radiation, immunocompromised state, history of prior psoralen and PUVA treatment, HIV infection, organ transplant, and hematological malignancies. The size of the tumor can be variable with local deep tissue invasion. It can also metastasize to regional lymph nodes or distant organ structures like the colon $[10,11]$.

Patients may present with complaints of rapidly growing, firm, non-tender, and red to violaceous nodules localized on the sunexposed skin, most commonly the head and neck region. It can be red, blue, purple, or skin-colored and sometimes presents with ulceration and crusting [12]. The diagnosis of MCC is based on findings on light microscopy, electron microscopy, and immunohistochemistry. Microscopically, the tumor is mainly centered in the dermis or sometimes in the subcutaneous tissue, without the involvement of the overlying epidermis. The tumor cells are composed of strands or nests of round, blue cells containing large basophilic nuclei [13]. MCC can be distinguished from other skin malignancies by immunostaining on immunohistochemistry. Mainly, it stains positive for epithelial markers like CK20 in a peculiar "dot-like" fashion and stains negative for epithelial markers like LCA, S-100, CK7, and TTF-1 [14] but it can be positive for various neuroendocrine markers like chromogranin, synaptophysin, calcitonin, vasoactive intestinal peptide, substance $\mathrm{P}$, ACTH, pancreatic polypeptide, somatostatin receptor, NSE, neurofilament, and CD $56[15,16]$.

Because of the clinical similarity on the initial presentations, MCC can be confused as benign lesions of skin like lipoma, nodule, cysts, pyogenic granuloma, and misdiagnosed and missed until the advanced stage. Any patient with suspicious lesions should be appropriately evaluated with a thorough history, proper skin examination, biopsy with immunohistochemistry of the affected areas to rule out MCC. The patient must undergo scanning of the body for the staging of cancer $[17,18]$.

Treatment of the MCC depends upon the stage of cancer. Any localized disease can be treated with wide local excision of the tumor and adjuvant local radiation therapy. In patients with lymph node involvement, excision of the adjacent lymph nodes can be done by radiation therapy [19]. Still, in those in whom lymph nodes cannot be resected, treatment with neoadjuvant radiochemotherapy followed by lymph node dissection can be considered [20]. MCC is highly radiosensitive; hence any patient with metastatic MCC should be treated with a combination of palliative chemotherapy and radiotherapy. In patients with cutaneous deposits or bone and brain metastases radiotherapy, about $50 \mathrm{~Gy}$ can be used as palliative therapy [21]. Despite treatment, any patient with MCC is at high risk of recurrence of disease, and the overall prognosis is generally less than 36 months. Recently treatment with Immunotherapy (antibodies targeting the programmed cell death protein 1/programmed cell death ligand 1) has shown a promising effect in patients with Stage III or IV MCC like in our patient [22]. Our patient was treated with pembrolizumab, an immune checkpoint inhibitor, every three weeks and completed 22 cycles of treatment without any complication.

Pembrolizumab, an anti-programmed death receptor-1 monoclonal antibody, and avelumab, an anti-programmed death ligand-1 monoclonal antibody, are currently approved for treating metastatic Merkel cell carcinoma. These agents allow T-cell activation and induce an antitumor response [22]. Patients should be followed up every 3-4 months for complete examination for 2-3 years as MCC has a very high chance of recurrence despite adequate treatment. MCC is found to have a poor prognosis because of the aggressive nature and late diagnosis. Patients of MCC with lymph node involvement, tumor size $>2 \mathrm{~cm}$, age $>60$ years, immunosuppression, the involvement of lower extremities, and metastasis to gastrointestinal organs and pleura are considered to have a poor prognosis [22].

\section{Conclusion}

In this case report, we report Merkle cell carcinoma's metastasis 
to the intestine, which is a very rare presentation. It is well known that MCC is aggressive cancer with a poor prognosis, but the addition of immunotherapy has shown better survival and quality of life like in our patient.

\section{References}

1. PW Harms. On behalf of the International Workshop on Merkel Cell Carcinoma Research (IWMCC) Working Group, KL Harms et al. "The biology and treatment of Merkel cell carcinoma: current understanding and research priorities". Nature Reviews Clinical Oncology. 2018; 15: 763-776.

2. TL Fitzgerald, S Dennis, SD Kachare, NA Vohra, JH Wong and EE Zervos. "Dramatic increase in the incidence and mortality from Merkel cell carcinoma in the United States". The American Surgeon. 2015; 81: 802-806.

3. Fitzgerald TL, Dennis S, Kachare SD, Vohra NA, Wong JH, Zervos EE Dramatic increase in the incidence and mortality from Merkel cell carcinoma in the United States. Am Surg. 2015; 81: 802-806.

4. M Heath, N Jaimes, B Lemos, et al. "Clinical characteristics of Merkel cell carcinoma at diagnosis in 195 patients: the AEIOU features". Journal of the American Academy of Dermatology. 2008; 58: 375-381.

5. Kempf W, Mertz KD, Hofbauer GF, Tinguely M. Skin cancer in organ transplant recipients. Pathobiology. 2013; 80: 302-309.

6. D Schadendorf, C Lebbé, A zur Hausen et al. "Merkel cell carcinoma: epidemiology, prognosis, therapy and unmet medical needs". European Journal of Cancer. 2017; 71: 53-69.

7. G-S Huang, W-C Chang, H-S Lee, JAM Taylor, T-Y Cheng and C-Y Chen "Merkel cell carcinoma arising from the subcutaneous fat of the arm with intact skin". Dermatologic Surgery. 2005; 31: 717-719.

8. Toker C. Trabecular carcinoma of the skin. Arch Dermatol. 1972; 105: 107110.

9. Dinh V, Feun L, Elgart G, Savaraj N. Merkel cell carcinomas. Hematol Oncol Clin North Am. 2007; 21: 527-544.

10. JJ Goedert and The Rockville Merkel Cell Carcinoma Group. "Merkel cell carcinoma: recent progress and current priorities on etiology, pathogenesis, and clinical management". J Clin Oncol. 2009; 27: 4021-4026.

11. RK Gandhi, AS Rosenberg and SC Somach. "Merkel cell polyomavirus: an update". Journal of Cutaneous Pathology. 2009; 36: 1327-1329.
12. Rosai J. Rosai and Ackerman's Surgical Pathology. Mosby. 2004; 1: 177179.

13. O Bayrou, MF Avril, P Charpentier, B Caillou, JC Guillaume and M Prade. "Primary neuroendocrine carcinoma of the skin. Clinicopathologic study of 18 cases". Journal of the American Academy of Dermatology. 1991; 24: 198207.

14. P Nghiem and N Jaimes. "Chapter 120: Merkel cell carcinoma". In Fitzpatrick's Dermatology in General Medicine, K Wolff, S Katz, L Goldsmith, B Gilchrest, D Leffell and A Paller, Eds., chapter 120, McGraw-Hill, New York, NY, USA $7^{\text {th }}$ edition. 2008

15. Sidhu GS, Feiner TJ, Mullins JD, Schaefler K, Schultenover SJ. Merkel cell Neoplasms. Histology, electron microscopy, biology and histogenesis. Am J Dermatopathol. 1980; 2: 101-119.

16. Wick MR, Scheithauer BW, Kovacs K. Neuron-specific enolase in neuroendocrine tumours of the thymus bronchus and skin. Am J Clin Pathol. 1983; 79: 703-707.

17. Brinkschmidt $C$, Stolze $P$, Fahrenkamp AG, Hundeiker M, Fischer-Colbrie $\mathrm{R}$, Zelger $\mathrm{B}$, et al. Immunohistochemical demonstration of chromogranin $A$, chromogranin B, and secretoneurin in Merkel cell carcinoma of the skin. An immunohistochemical study on 18 cases suggesting two types of Merkel cell carcinoma. Appl Immunohistochem. 1995; 3: 37-44.

18. Nguyen B, McCullough A. Imaging of Merkel cell carcinoma. Radiographics. 2002; 22: 367-337.

19. Nguyen BD. Positron emission tomographic imaging of Merkel cell carcinoma. Clin Nucl Med. 2002; 27: 922.

20. M Papamichail, I Nikolaidis, N Nikolaidis, et al. "Merkel cell carcinoma of the upper extremity: case report and an update". World Journal of Surgical Oncology. 2008; 6: 32.

21. Guihard S, Noël G. Merkel cell carcinoma, role of radiotherapy and literature r19. Pectasides D, Pectasides M, Economopoulos T. Merkel cell cancer of the skin. Ann Oncol. 2006; 24: 1489-1495.

22. Paulson KG, Bhatia S. Advances in Immunotherapy for Metastatic Merkel Cell Carcinoma: A Clinician's Guide. J Natl Compr Canc Netw. 2018; 16: $782-790$ 